\title{
Modelos reológicos asociados al néctar mix papaya - piña
}

\author{
Angel Sobrado Gómez \\ asobrado@unheval.edu.pe \\ Orcid: 0000-0001-7296-9345 \\ Docente de la Universidad Nacional Hermilio Valdizan de Huánuco
}

\section{RESUMEN}

Objetivo: se tuvo como objetivo Determinar los modelos reológicos asociados al néctar mix de papaya - piña. Método: el método de investigacion se realizó haciendo uso del reómetro rotacional Rheolab QC, donde se midieron las variables de la ecuación reológico que vienen a ser el esfuerzo de corte y la velocidad de deformación. Las corridas se hicieron a las temperaturas de $20^{\circ} \mathrm{C}, 30^{\circ} \mathrm{C}, 40^{\circ} \mathrm{C}, 50^{\circ} \mathrm{C}, 60^{\circ} \mathrm{C}, 70^{\circ} \mathrm{C}$ a fin de evaluar el efecto de la temperatura sobra la viscosidad y encontrar una energía de activación promedio. La muestra de néctar utilizada es de $50 \%$ de zumo de papaya y $50 \%$ de zumo de piña. Resultados el logro del trabajo fueron las características de un fluido plástico real, con un umbral de esfuerzo $=0.5667 \mathrm{~Pa}$, índice de consistencia $=$ 0.2185 Pa.sn e índice de flujo $=0.6401$ a la temperatura de $20^{\circ} \mathrm{C}$, así como una viscosidad aparente $=0.0473 \mathrm{~Pa}$.s y energía de activación $=3473.9 \mathrm{~J} / \mathrm{mol}$ para el rango de temperatura de 20 a $70{ }^{\circ} \mathrm{C}$ a una velocidad de corte de 5 s-1. Conclusiones: el Néctar mix papaya - piña presenta comportamiento reológico de un fluido plástico real, siendo el modelo de Herschel Bulkley, dado por la ecuación: $\tau=\tau_{0}+(\mu \gamma)^{1 / m}$, con un umbral de esfuerzo $=0.5667 \mathrm{~Pa}$, índice de consistencia $=0.2185 \mathrm{~Pa} \cdot \mathrm{s}^{\mathrm{n}}$ e índice de flujo $=$ 0.6401 a la temperatura de $20^{\circ} \mathrm{C}$, así como una viscosidad aparente $=0.0473 \mathrm{~Pa}$.s y energía de activación $=3.7439 \mathrm{KJ} / \mathrm{mol}$ para el rango de temperatura de 20 a $70{ }^{\circ} \mathrm{C}$ a una velocidad de corte de $5 \mathrm{~s}^{-1}$, y con los valores estadísticos: coeficiente de correlación $\mathrm{R}^{2}$ $=0.999238$ y varianza $S^{2}=0.00157717$ para un nivel de confianza del $95 \%$, el modelo de representación óptima para este tipo de fluido en referencia a los demás modelos estudiados en esta tesis.

Palabras clave: modelos reológicos; néctar mix papaya - piña; ecuación 


\title{
Rheological models associated with papaya-pineapple nectar mix
}

\begin{abstract}
Objective: The nectar aimed at Determining rheological correlated models itself mix of papaya - pineapple. Method: The method of investigation came true making use of the rotational rheometer Rheolab QC, where they measured the variables of the rheological equation that serve to be court's effort and the velocity of deformation. Races plucked up to the temperatures $20 \mathrm{C}, 30 \mathrm{C}, 40 \mathrm{C}, 50 \mathrm{C}, 60 \mathrm{C}, 70$ the viscosity and finding an energy of average activation is more than enough $\mathrm{C}$ in order to evaluate the effect of temperature. The sign of nectar utilized comes from $50 \%$ of papaya juice and $50 \%$ of pineapple juice. Results the achievement of work 0,5667 Pa, index of consistency were the characteristics of a fluid real plastic with a doorstep of effort, 0,2185 Pa.sn and index of flow 0,6401 to the temperature of $20 \mathrm{C}$, as well as an apparent viscosity 0,0473 $\mathrm{Pa} . \mathrm{s}$ and energy of activation $3473,9 \mathrm{~J}$ mole for the range of temperature of 20 to $70 \mathrm{C}$ to court's velocity of $5 \mathrm{~s}$ 1. Findings: The Nectar mix papaya - the pineapple presents rheological behavior of a fluid real plastic, being Herschel Bulkley's model, die for the equation:, with a doorstep of effort 0,5667 $\mathrm{Pa}$, index of consistency 0,2185 Pa.sn and index of flow 0,6401 to the temperature of $20 \mathrm{C}$, as well as an apparent viscosity 0,0473 Pa.s and energy of activation 3,7439 KJ mole for the range of temperature of 20 to $70 \mathrm{C}$ to court's velocity of $5 \mathrm{~s}^{1}$, and with the statistical moral values: Correlation coefficient R2 0,999238 and variance S2 0,00157717 for the 95\%'s confidence level, the model of optimal performance for this type of fluid in regard to the rest of the models gone into in this thesis.
\end{abstract}

Keywords: rheology; nectar mix papaya - pineapple; equation

Artículo recibido: 10. Junio. 2021 Aceptado para publicación: 16. Julio. 2021 Correspondencia: asobrado@unheval.edu.pe

Conflictos de Interés: Ninguna que declarar 


\section{INTRODUCCION}

En la actualidad existen herramientas informáticas sofisticadas que nos permiten desarrollar expresiones matemáticas complejas en las que intervienen iteraciones extensivas, múltiples variables y manejo de gráficos multidimensionales. Gracias a este avance, los problemas que surgen entorno al cálculo se ven reducidos, dando lugar a la imaginación del investigador para explorar nuevos niveles de tratamientos en un determinado fenómeno. Basándonos en la teoría de fenómenos de transporte, planteamos la búsqueda del modelo que explique la reología del zumo mix papaya piña. Existe una variedad finita de estos modelos, entre los cuales se encuentra el modelo de Herschel Bulkley, Robertson Stiff, Ellis de Haven, entre otros modelos de investigadores contemporáneos. De esta manera haciendo uso de un reómetro mediremos las variables de la ecuación reológica que vienen a ser el esfuerzo de corte y la velocidad de deformación del flujo en diferentes niveles de tratamiento al zumo de mango. Este aporte permitirá la predicción del flujo del zumo (índice de consistencia, índice de comportamiento al flujo) bajo diferentes condiciones de concentración y temperatura, lo cual resulta de utilidad en el cálculo de potencias en el diseño de un sistema de bombeo, sistema de agitación, reactores con agitación, en el diseño de tuberías y en el control de calidad de productos.

\section{METODOLOGÍA.}

\section{Lugar de ejecución}

Extracción de zumos de papaya y piña, muestras obtenidas en laboratorio de procesos agroindustriales de la Universidad Nacional Hermilio Valdizán. Los ensayos reológicos fueron desarrollados en el laboratorio de investigación de la facultad de ingeniería química de la universidad nacional del callao.

\section{Tipo de investigacion}

Los tipos de investigación que se realizan en acuerdo al proyecto de esta tesis son:

Por su finalidad es de tipo exploratorio, puesto que busca identificar nuevos rumbos para investigación en campos de conocimiento no estudiadas o poco estudiados.

Por su diseño interpretativo es experimental por que permitirá manipular el factor causal para determinar el efecto deseado.

Por el énfasis de la naturaleza de los datos manejados es del tipo cuantitativo por que los variables de la investigación son cuantitativas 


\section{Método de investigacion}

Correlacional: Debido a que la utilidad de este tipo de investigación es saber cómo se puede comportar un concepto o variable conociendo el comportamiento de otra u otras variables relacionadas.

Transversal: Debido a que se determinará los parámetros y modelos matemáticos en un tiempo determinado interesando estudiar el problema en ese momento.

Experimental: Ya que se realizará un conjunto de actividades metódicas y técnicas para recabar la información y datos necesarios sobre el tema a investigar y el problema a resolver

\section{Diseño de investigacion}

El diseño de la presente investigación consiste de tres momentos
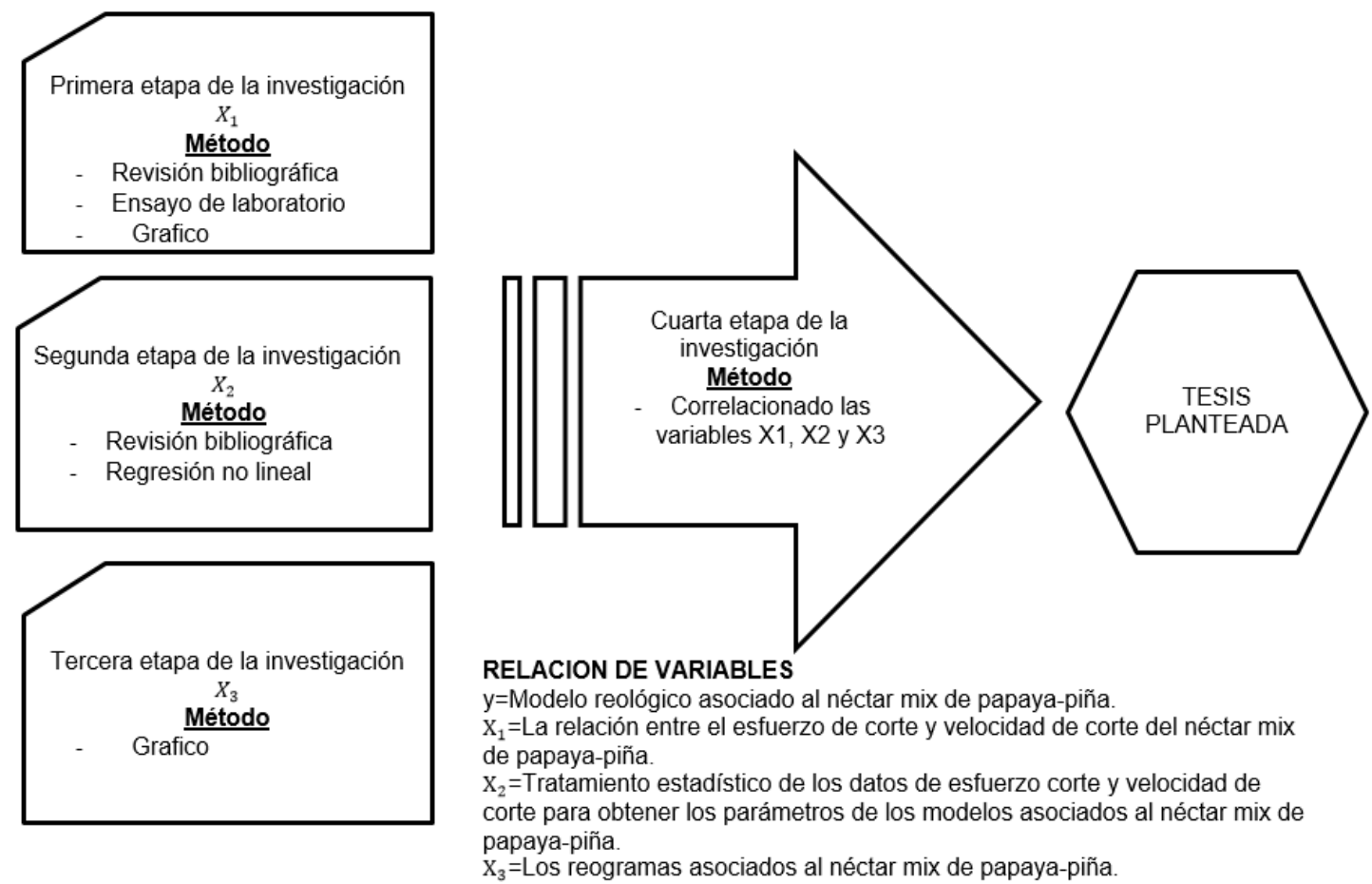

\section{Población}

Lote de néctar mix papaya-piña producido en la sala de procesos agroindustriales de la UNHEVAL.

\section{Muestra}

Diez botellas de $300 \mathrm{~mL}$ de néctar mix papaya - piña con $14^{\circ}$ brix, volumen necesario para las mediciones en el reómetro. 


\section{Periodo de investigacion}

La investigacion se desarrolló durante el periodo abril del 2016 hasta diciembre 2018

\section{Técnicas e Instrumentos para la recolección de la informacion}

\section{Técnicas}

Mediante la experimentación con el reómetro rotacional para la obtención de los datos de corte y velocidad de corte del zumo mix papaya piña, la cual se detalla.

Extracción de zumos de papaya y piña, muestras obtenidas en un labotorio de sala de procesos ogroindustriales de la UNHEVAL

Se seleccionó $70 \mathrm{ml}$ de muestra de Zumo de papaya y piña de $14^{\circ}$ brix para las corridas experimentales.

Cada muestra a analizar se puso en un vaso precipitado de $100 \mathrm{ml}$, se preparó el equipo (reómetro) las diferentes corridas a realizar

Se colocó la muestra de zumo mix papaya-piña en el contenedor (Cup) del reómetro, se controló la temperatura con un chaleco térmico acoplado al cup y se fijó la velocidad de rotación a la cual fue analizada la muestra.

Se realizó la corrida experimental a las temperaturas de $20,3040,50,60$ y $70{ }^{\circ} \mathrm{C}$ y cada una por duplicado para obtener puntos exactos.

El reómetro dio como resultados los valores del esfuerzo de corte y la velocidad de corte de la muestra a la temperatura de experimentación.

Con los datos obtenidos del reómetro se realizó la regresión de los modelos reológicos y el análisis estadístico para encontrar el modelo de fluido no newtoniano que se asocia al comportamiento del néctar mix de papaya y piña, y se obtuvo la vez los diferentes reogramas asociados al néctar mix de papaya y piña.

Así mismo, la técnica de regresión de mínimos cuadrados para modelar el comportamiento reológico de dicho fluido

\section{Instrumentos}

El principal instrumento de la investigación fue el reómetro rotacional Rheolab QC, ubicado en el Laboratorio de Investigación de la Facultad de Ingeniería Química de la Universidad Nacional del Callao, así como el software Minitab para los análisis estadísticos. 


\section{RESULTADOS}

\section{Resultados descriptivos}

Se evaluó el comportamiento reológico del néctar mix papaya-piña de 14 grados brix a las temperaturas $20,30,40,50,60$ y $70^{\circ} \mathrm{C}$.

Tabla 1 datos reologicos del néctar mix papaya piña

\begin{tabular}{ccccccccccccc}
\hline \multicolumn{2}{c}{$\mathbf{T}\left({ }^{\circ} \mathbf{C}\right)=\mathbf{2 0}$} & \multicolumn{3}{c}{$\mathbf{T}\left({ }^{\circ} \mathbf{C}\right)=\mathbf{3 0}$} & \multicolumn{2}{c}{$\mathbf{T}\left({ }^{\circ} \mathbf{C}\right)=\mathbf{4 0}$} & \multicolumn{2}{c}{$\mathbf{T}\left({ }^{\circ} \mathbf{C}\right)=\mathbf{5 0}$} & \multicolumn{2}{c}{$\mathbf{T}\left({ }^{\circ} \mathbf{C}\right)=\mathbf{6 0}$} & \multicolumn{2}{c}{$\mathbf{T}\left({ }^{\circ} \mathbf{C}\right)=\mathbf{7 0}$} \\
\hline$\square(1 / \mathrm{s})$ & $\square(\mathrm{Pa})$ & $\square(1 / \mathrm{s})$ & $\square(\mathrm{Pa})$ & $\square(1 / \mathrm{s})$ & $\square(\mathrm{Pa})$ & $\square(1 / \mathrm{s})$ & $\square(\mathrm{Pa})$ & $\square(1 / \mathrm{s})$ & $\square(\mathrm{Pa})$ & $\square(1 / \mathrm{s})$ & $\square(\mathrm{Pa})$ \\
3.21 & 1 & 5.11 & 1 & 4.57 & 1 & 1.36 & 1 & 4.6 & 1 & 1.29 & 1 \\
8.72 & 1.5 & 9.15 & 1.5 & 7.51 & 1.5 & 5.24 & 1.5 & 10.8 & 1.5 & 3.59 & 1.5 \\
19.2 & 1.99 & 16.1 & 1.99 & 14.8 & 1.99 & 13.7 & 1.99 & 19 & 1.99 & 11.9 & 1.99 \\
30.4 & 2.49 & 25 & 2.49 & 24.1 & 2.49 & 23.8 & 2.49 & 30.3 & 2.49 & 21.7 & 2.49 \\
42.5 & 2.99 & 35.8 & 2.99 & 36.3 & 2.99 & 36.6 & 2.99 & 43.9 & 2.99 & 35.5 & 2.99 \\
57.5 & 3.49 & 49.1 & 3.49 & 51.7 & 3.49 & 52.7 & 3.49 & 60.7 & 3.49 & 52.5 & 3.49 \\
73.4 & 3.98 & 65.4 & 3.98 & 69.2 & 3.98 & 71.7 & 3.98 & 80.9 & 3.98 & 72.2 & 3.98 \\
90.5 & 4.48 & 83.8 & 4.48 & 89.4 & 4.48 & 93 & 4.48 & - & - & 94.8 & 4.48 \\
\hline
\end{tabular}

Figura 1. Reograma experimantal de esfuerzo de corte

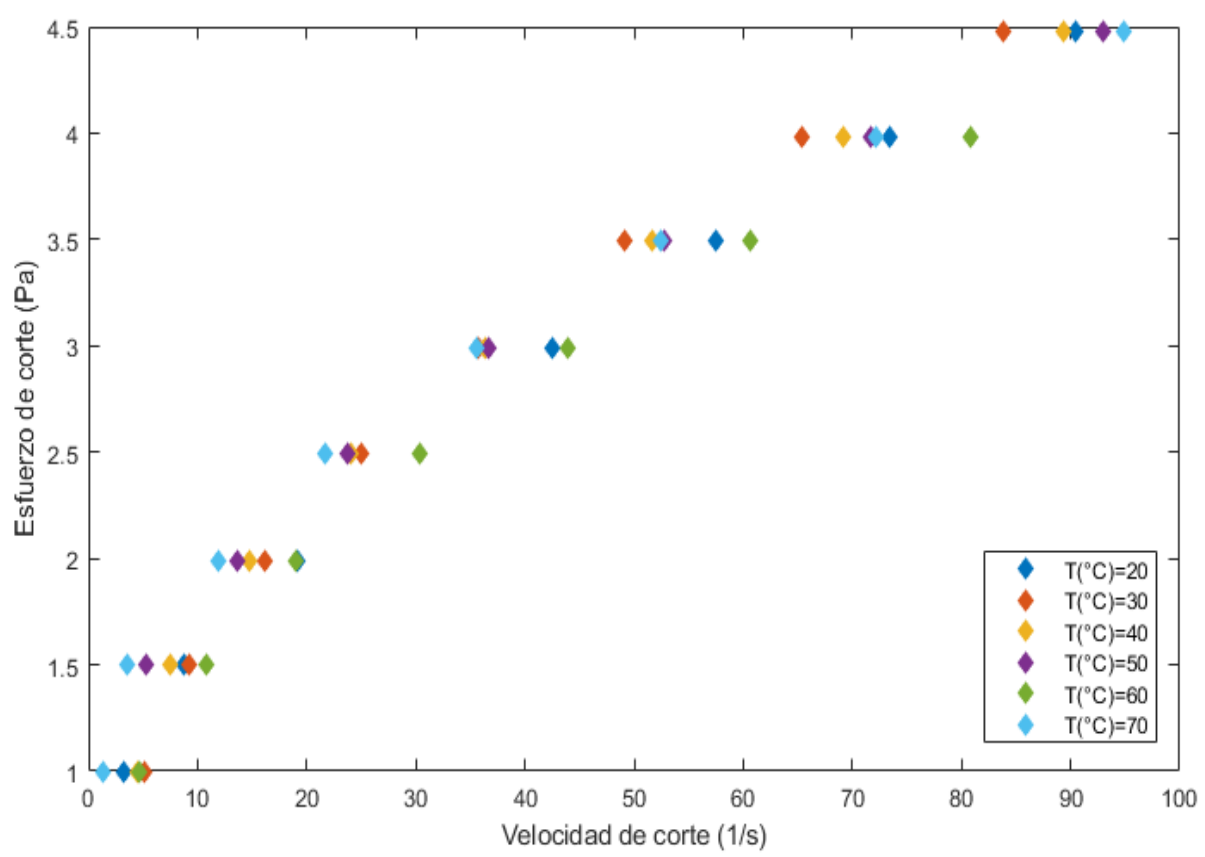

Resultados inferenciales: regresión minimos cuadrados aplicados a los modelos reologicos. 
Modelo Ostwald de Waele aplicado al Néctar mix papaya - piña

Tabla 2. Modelo Ostwald waele

\begin{tabular}{ccc}
\hline Modelo reológico & Ecuación de regresión & Parámetros \\
\hline$\tau=k \gamma^{n}$ & $y=k x^{n}$ & $k, n$ \\
\hline
\end{tabular}

Tabla 3. Parametros del modelo Ostwald de Waele

\begin{tabular}{ccccc}
\hline $\boldsymbol{T}\left({ }^{\circ} \boldsymbol{C}\right)$ & $\boldsymbol{K}$ & $\boldsymbol{n}$ & $\boldsymbol{R} 2$ & \multicolumn{1}{c}{ Varianza } \\
\hline 20 & 0.4924231 & 0.4856606 & 0.9949203 & 0.0087882 \\
30 & 0.4882903 & 0.5026152 & 0.9982909 & 0.0029568 \\
40 & 0.5664577 & 0.4610237 & 0.9973894 & 0.0045164 \\
50 & 0.7467011 & 0.3908642 & 0.9944159 & 0.0096609 \\
60 & 0.4802908 & 0.4822769 & 0.9998887 & 0.000154 \\
70 & 0.8470324 & 0.3607884 & 0.9940178 & 0.0103496 \\
\hline
\end{tabular}

Figura 2. Ajuste del modelo Ostwald de waele

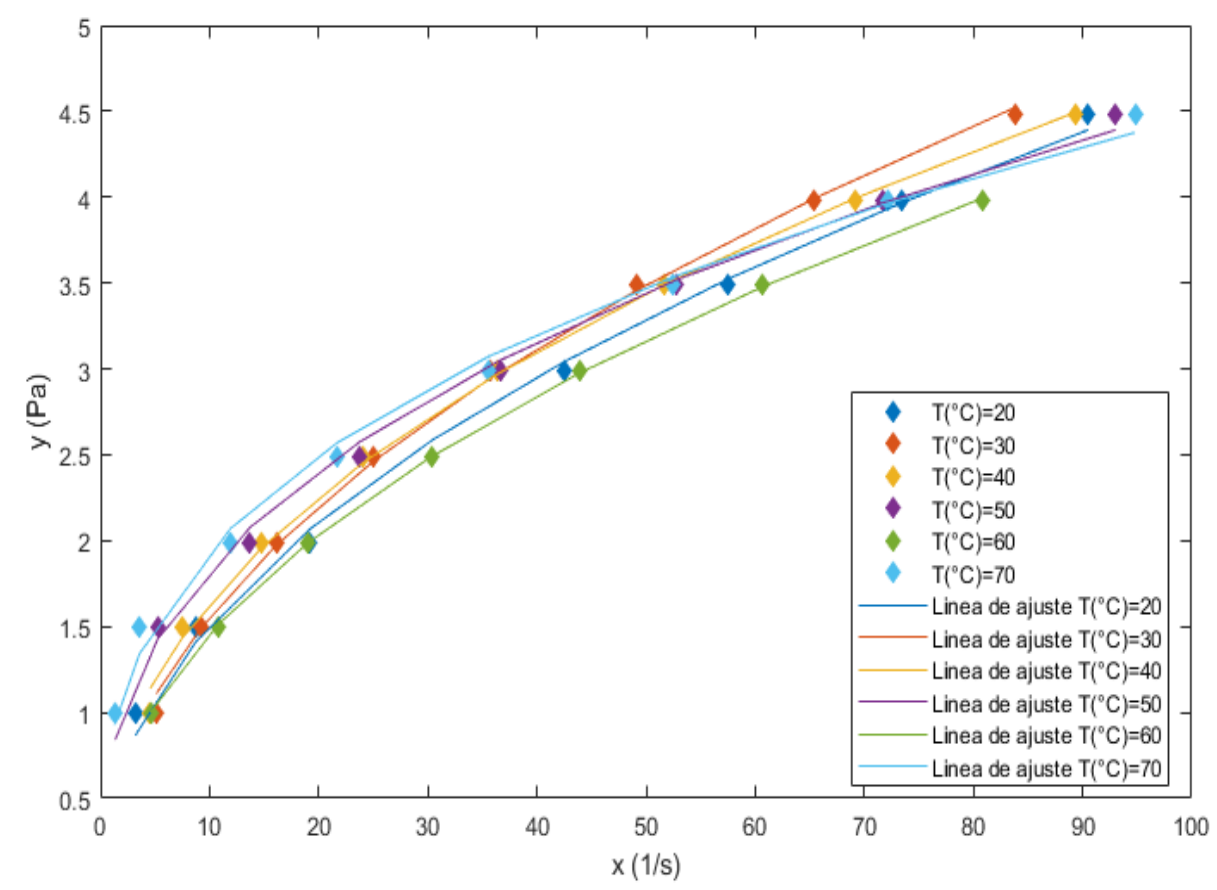


Modelo Robertson-Stiff aplicado al Nectar mix papaya-piña

Tabla 4. Modelo Robertson Stiff

\begin{tabular}{ccc}
\hline Modelo reológico & Ecuación de regresión & Parámetros \\
\hline$\tau=k\left(\gamma_{0}+\gamma\right)^{n}$ & $y=k\left(\gamma_{0}+x\right)^{n}$ & $k, \gamma_{0}, n$ \\
\hline
\end{tabular}

Tabla 5. Parametros del modelo Robertson-Stiff

\begin{tabular}{cccccc}
\hline $\boldsymbol{T}\left({ }^{\circ} \boldsymbol{C}\right)$ & $\boldsymbol{k}$ & $\dot{\boldsymbol{\gamma}}_{\mathbf{0}}$ & $\boldsymbol{n}$ & $\boldsymbol{R}^{2}$ & Varianza \\
\hline 20 & 0.3335971 & 4.085127 & 0.5702853 & 0.9992632 & 0.0015296 \\
30 & 0.5933372 & -1.920606 & 0.4588628 & 0.9998503 & 0.0003107 \\
40 & 0.6776517 & -1.770907 & 0.4207865 & 0.9988979 & 0.0022881 \\
50 & 0.5866367 & 2.180218 & 0.4455058 & 0.9993841 & 0.0012786 \\
60 & 0.4907059 & -0.2175217 & 0.4774614 & 0.9999082 & 0.0001589 \\
70 & 0.697068 & 1.652458 & 0.4053892 & 0.9973091 & 0.0055865 \\
\hline
\end{tabular}

Figura 3. ajuste del modelo Robertson

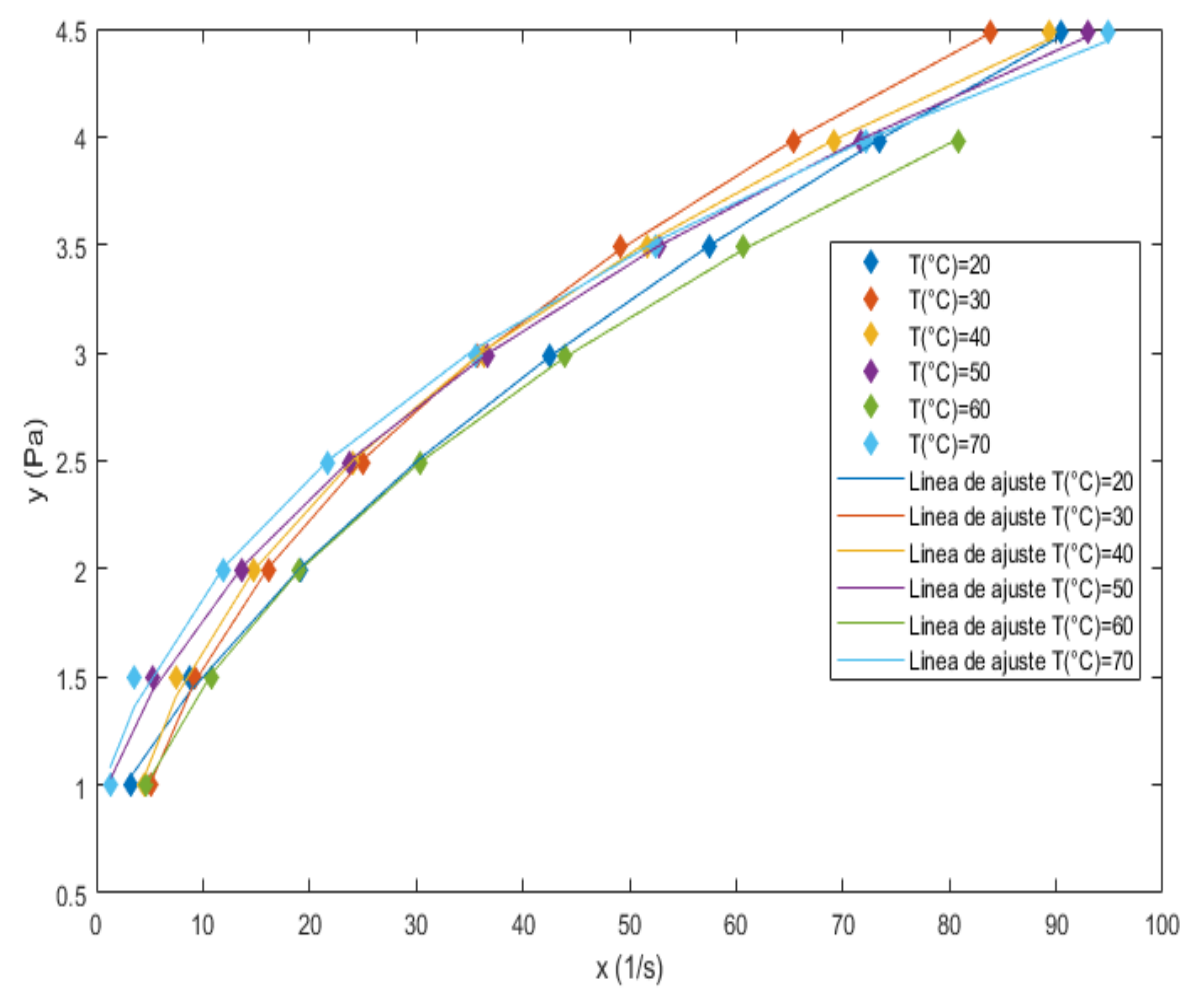


Modelo casson aplicado al Néctar mix papaya -piña

Tabla 6. Modelo Casson

\begin{tabular}{ccc}
\hline Modelo reológico & Ecuación de regresión & Parámetros \\
\hline$\tau=k\left(\gamma_{0}+\gamma\right)^{n}$ & $y=k\left(\gamma_{0}+x\right)^{n}$ & $k, \gamma_{0}, n$ \\
\hline
\end{tabular}

Tabla 7. Parametros del modelo Casson

\begin{tabular}{lllll}
\hline $\mathbf{2 0}$ & $\mathbf{0 . 6 3 7 4 2 8 5}$ & $\mathbf{0 . 1 3 9 9 4 3 1}$ & $\mathbf{0 . 9 9 8 0 7 1 2}$ & $\mathbf{0 . 0 0 3 3 3 7}$ \\
\hline 30 & 0.6279971 & 0.1489798 & 0.984238 & 0.0272693 \\
40 & 0.7333712 & 0.1372489 & 0.9816607 & 0.0317283 \\
50 & 0.8890413 & 0.1246006 & 0.9928565 & 0.0123587 \\
60 & 0.6148184 & 0.1381618 & 0.9895875 & 0.0144115 \\
70 & 0.974615 & 0.1189823 & 0.9888409 & 0.019306 \\
\hline
\end{tabular}

Figura 4. Ajustes del modelo Casson

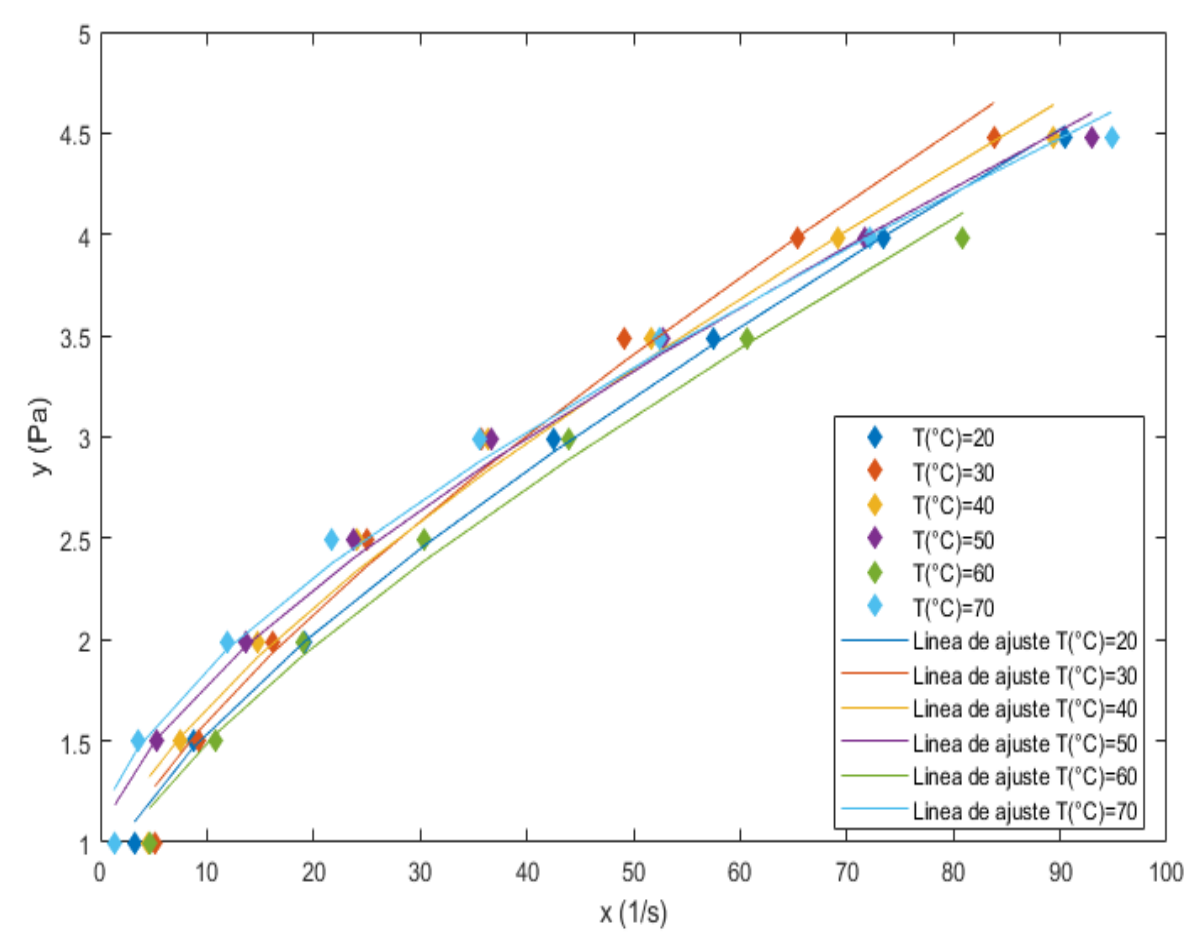




\section{DISCUSIONES}

A partir de los datos obtenidos, aceptamos la hipótesis general que establece que el néctar mix papaya piña presenta un comportamiento reológico del tipo plástico real, el cual queda representado adecuadamente por los modelos: Robertson Stiff, HerschelBulkley, Mizrahi-Berk y Sisko. De los cuales el modelo de Herschel-Bulkley para un intervalo de confianza de $95 \%$ obtiene un valor óptimo de $\mathrm{R}^{2}=0.999238$ y un valor mínimo de Varianza $S^{2}=0.00157717$. En las figuras 51 y 522 se observa los valores de $\mathrm{R}^{2}$ y varianza de los modelos representativos para el néctar mix.

Figura 5. Fluido plástico real

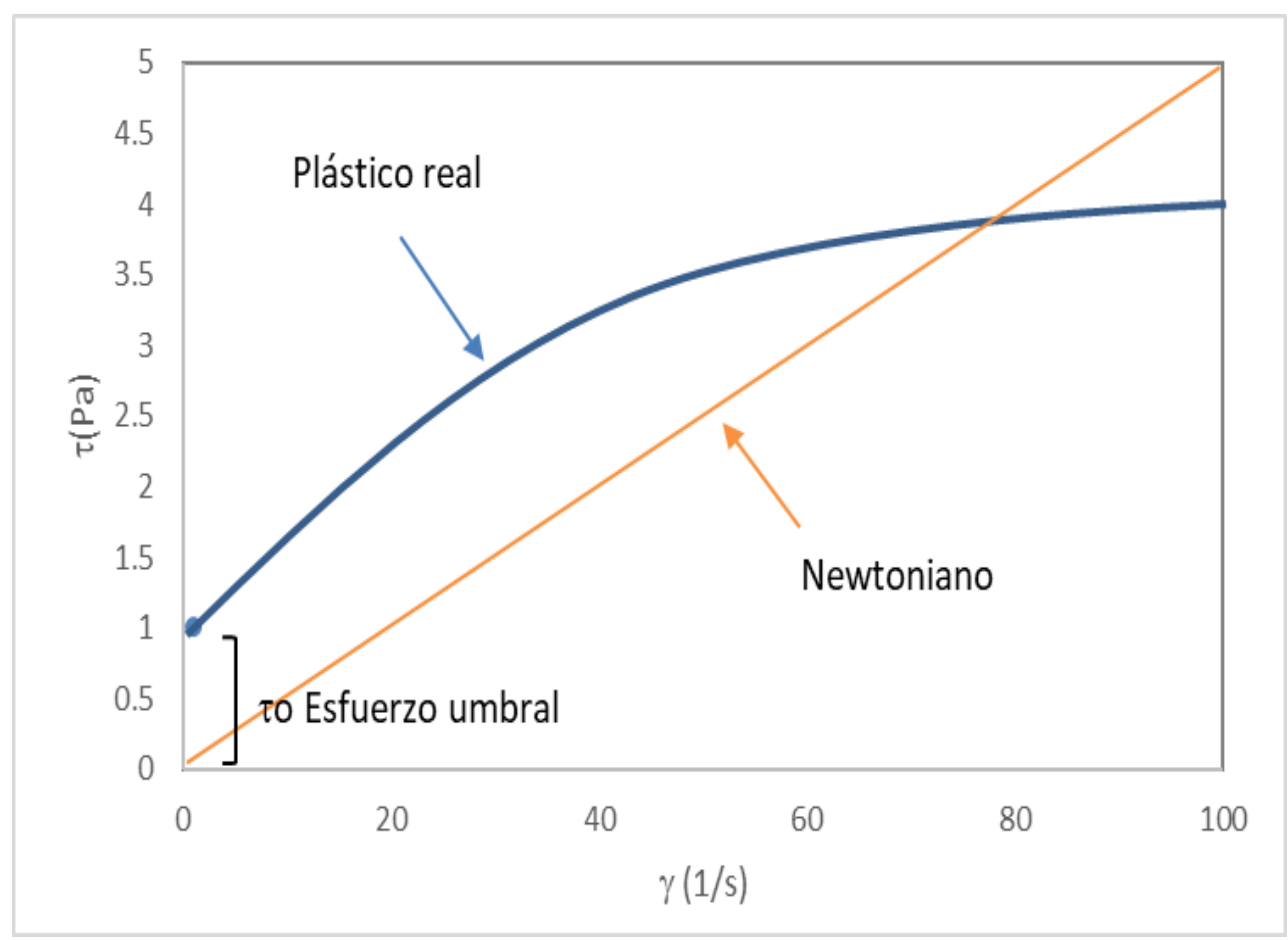

Tabla 8. Comportamiento No Ideal

\begin{tabular}{lcc}
\hline \multicolumn{1}{c}{ Modelo } & Ecuación & $\begin{array}{c}\text { Índice de comportamiento } \\
\text { promedio }\end{array}$ \\
\hline Herschel Bulkley & $\tau=\tau_{0}+(\mu \gamma)^{1 / m}$ & $1 / \mathrm{m}=0.48113414$ \\
Mizrahi Berk & $\tau^{0.5}=\tau_{0}^{0.5}+k \gamma^{n}$ & $\mathrm{n}=0.29993063$ \\
Sisko & $\tau=A \gamma+B \gamma^{n}$ & $\mathrm{n}=0.4172866$ \\
Robertson Stiff & $\tau=k\left(\gamma_{0}+\gamma\right)^{n}$ & $\mathrm{n}=0.4630485$ \\
\hline
\end{tabular}


Figura 6. Reograma esfuerzo velocidad y temperatura

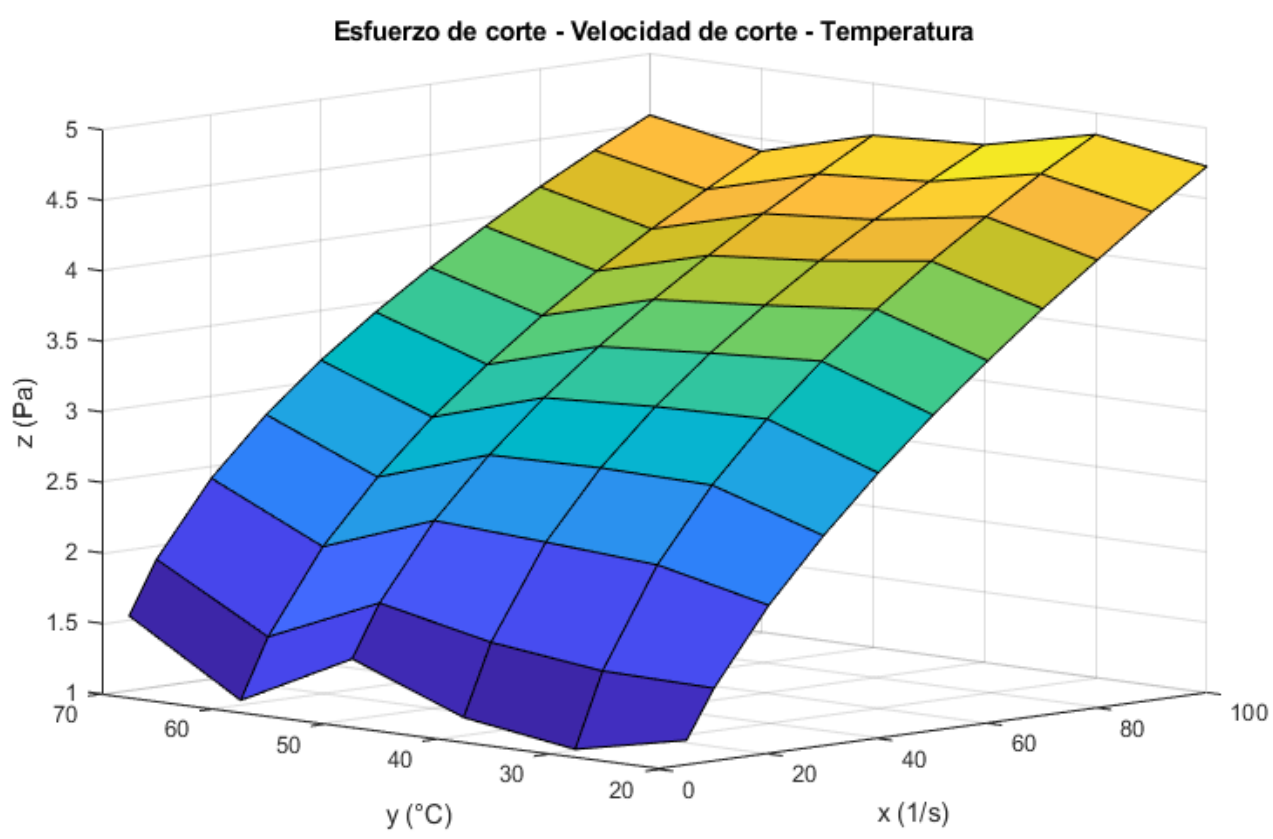

Figura 7. Reograma viscosidad, velocidad y esfuerzo

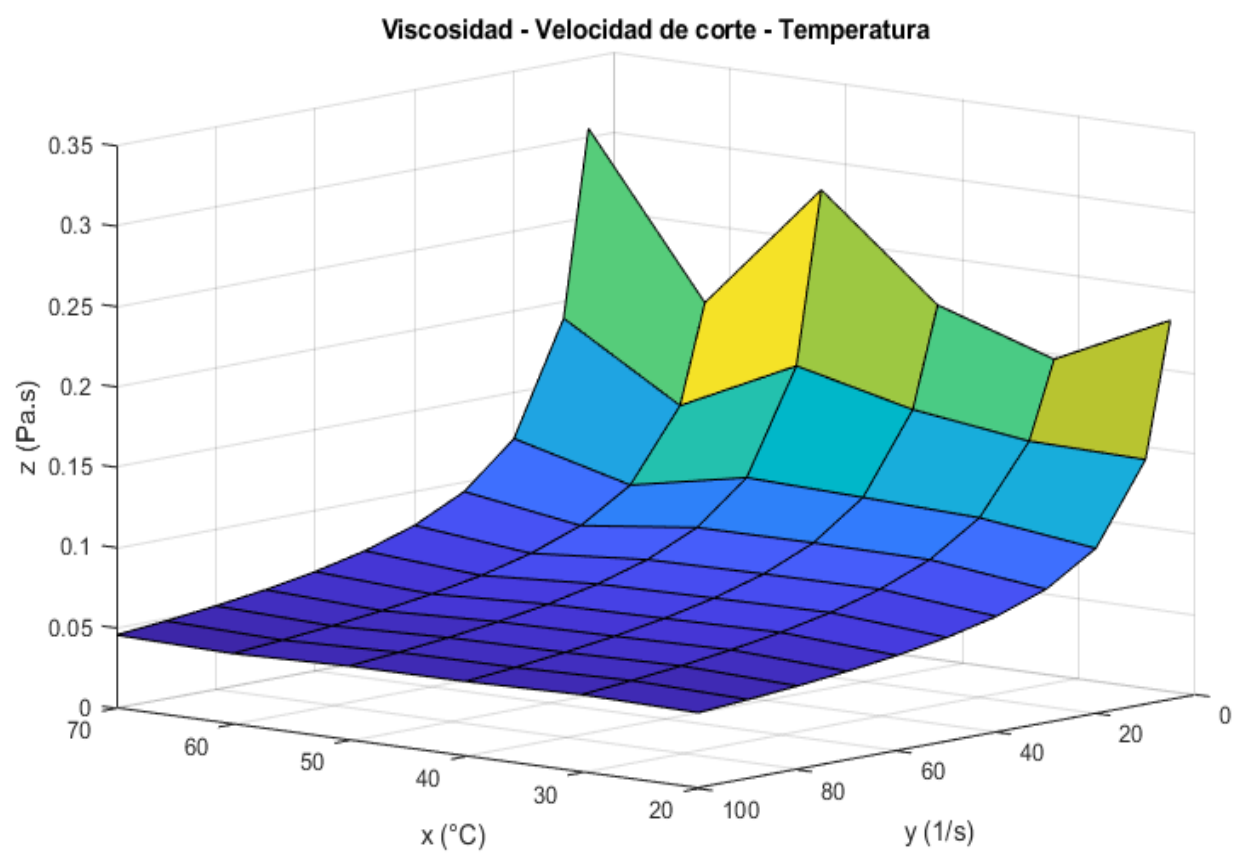

Ciencia Latina Revista Científica Multidisciplinar, Ciudad de México, México. 
Figura 8. Viscosidad, velocidad y temperatura

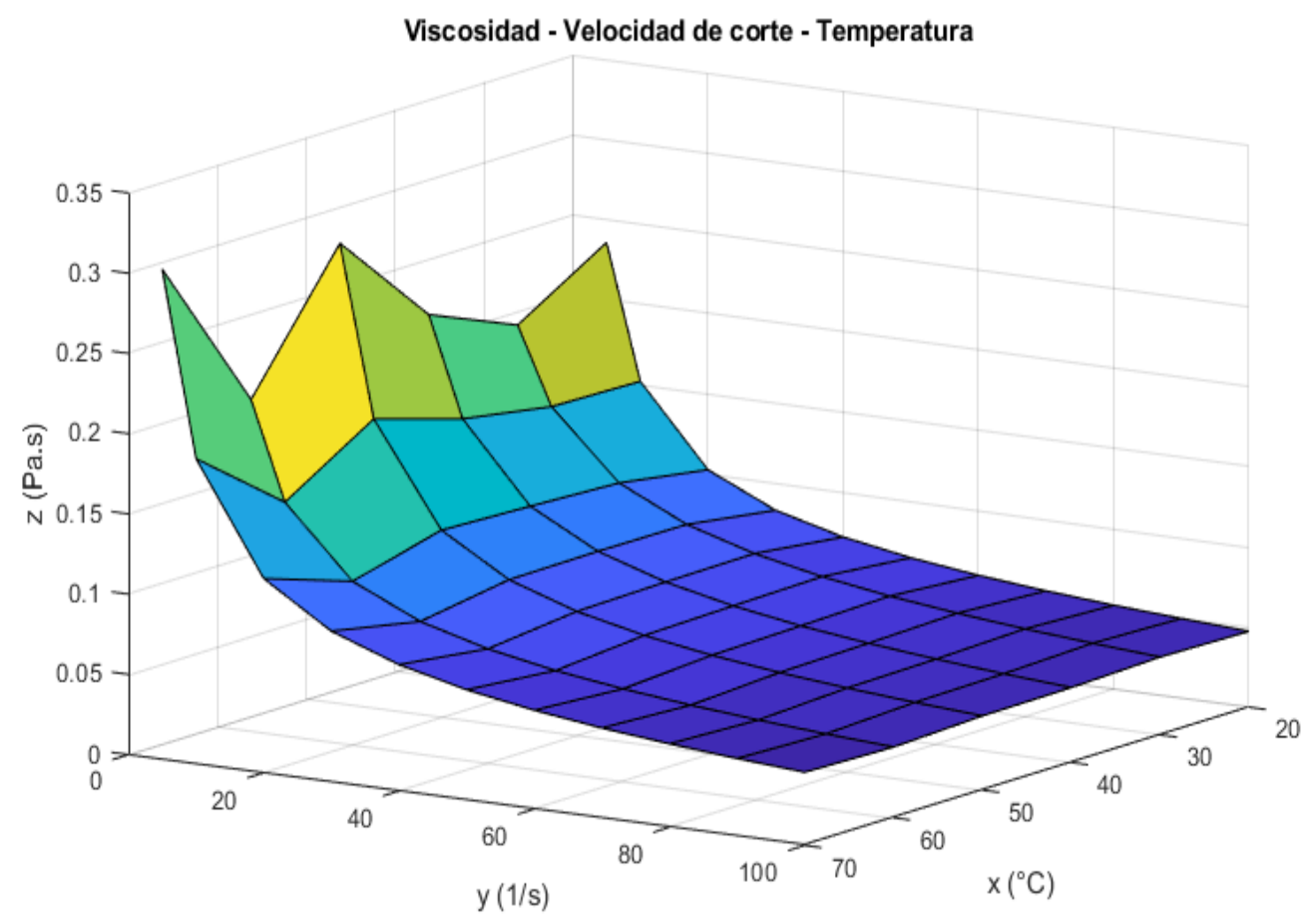

Del modelo de Herschel Bulkley, los resultados del índice de consistencia e índice de flujo promedio, $k=0.554867$ Pa.s $^{n}$ y $n=0.481134$ respectivamente, son valores característicos de un fluido con comportamiento plástico real.

Figura 9. Intervalos de $n$ del modelo Herschel Bulkley

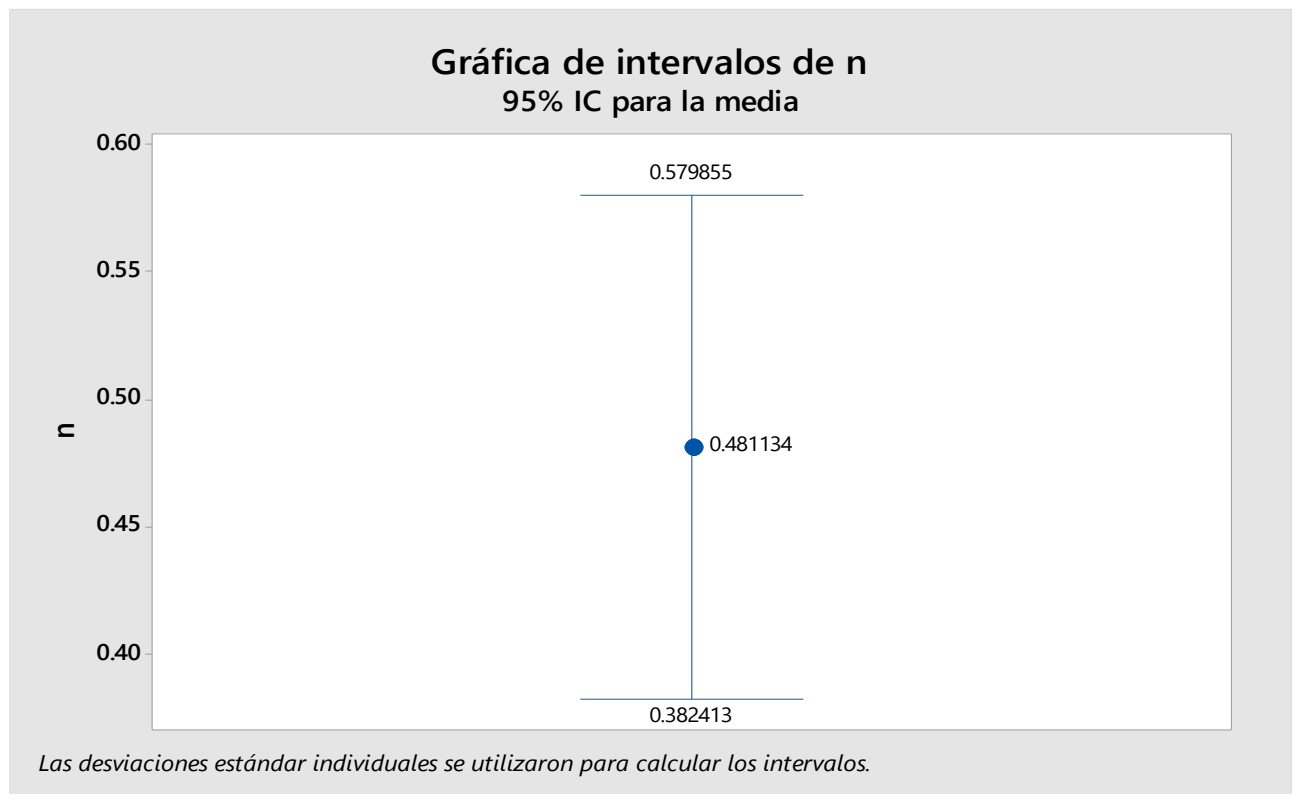




\section{Contrastación de los resultados con otros estudios similares}

Kawazoe y Cunha (2007) determinaron que el modelo de Herschel Bulkley fue el que mejor ajustó el comportamiento reológico de la fruta de jaboticaba. En el presente estudio, dicho modelo representa óptimamente, con un índice $\mathrm{R}^{2}=0.999238$, el comportamiento reológico del Mix papaya-piña. Dak y colaboradores (2006) evaluaron los parámetros reológicos para el jugo de mango con un reómetro rotacional el intervalo de temperaturas de 20 a $70^{\circ} \mathrm{C}$, determinando que el mejor ajuste se obtuvo con la ley de potencia. En el presente estudio, el modelo Ostwald de Waele (Ley de potencia) con un $\mathrm{R}^{2}=0.99665$ ajusta adecuadamente los datos reológicos del néctar mix papaya piña, siendo éste un modelo de mayor alcance para predecir el comportamiento reológico de jugos de fruta. Mossel y colaboradores (2000), determinaron la viscosidad de varias mieles australianas a tasas de deformación correspondientes a 1, 25 y $50 \mathrm{rpm}$ para un rango de temperatura entre $10-40{ }^{\circ} \mathrm{C}$. En el presente estudio, con el modelo de Herschel Bulkley se determinó la viscosidad del néctar mix papaya-piña para las velocidades de deformación desde 5 a $100 \mathrm{~s}^{-1}$ y el rango de temperaturas de 20 a $70^{\circ} \mathrm{C}$ demostrando que al aumentar la velocidad de deformación disminuye y al aumentar la temperatura aumenta hasta $\operatorname{los} 50^{\circ} \mathrm{C}(0.09875 \mathrm{~Pa} . \mathrm{s})$, decrece a los $60^{\circ} \mathrm{C}(0.08393 \mathrm{~Pa} . \mathrm{s})$ y vuelve a aumentar a los $70^{\circ} \mathrm{C}(0.10194 \mathrm{~Pa} . \mathrm{s})$.

Figura 10. Comportamiento de la viscosidad - velocidad de corte

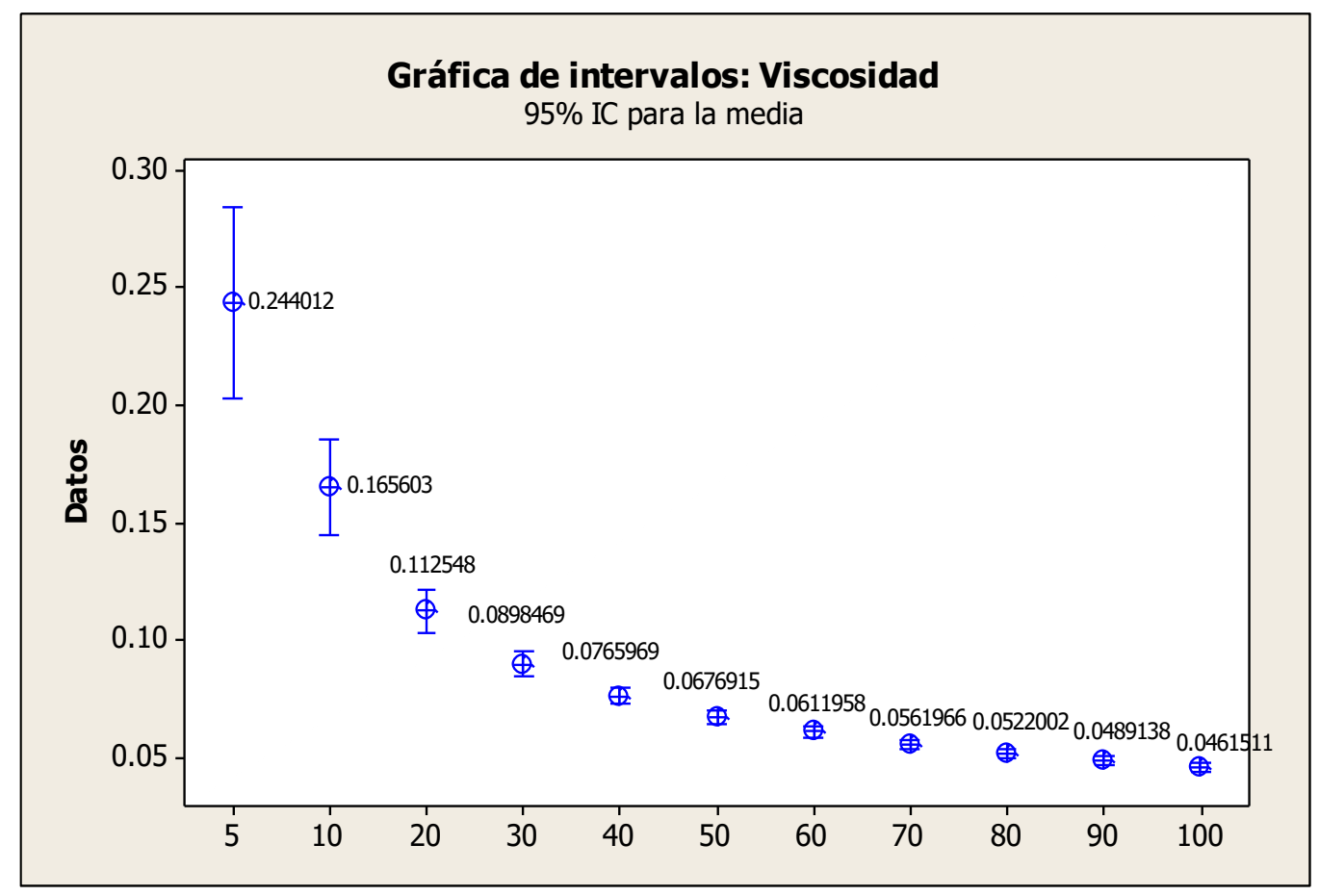


Figura 11. Comportamiento de la Viscosidad-Temperatura

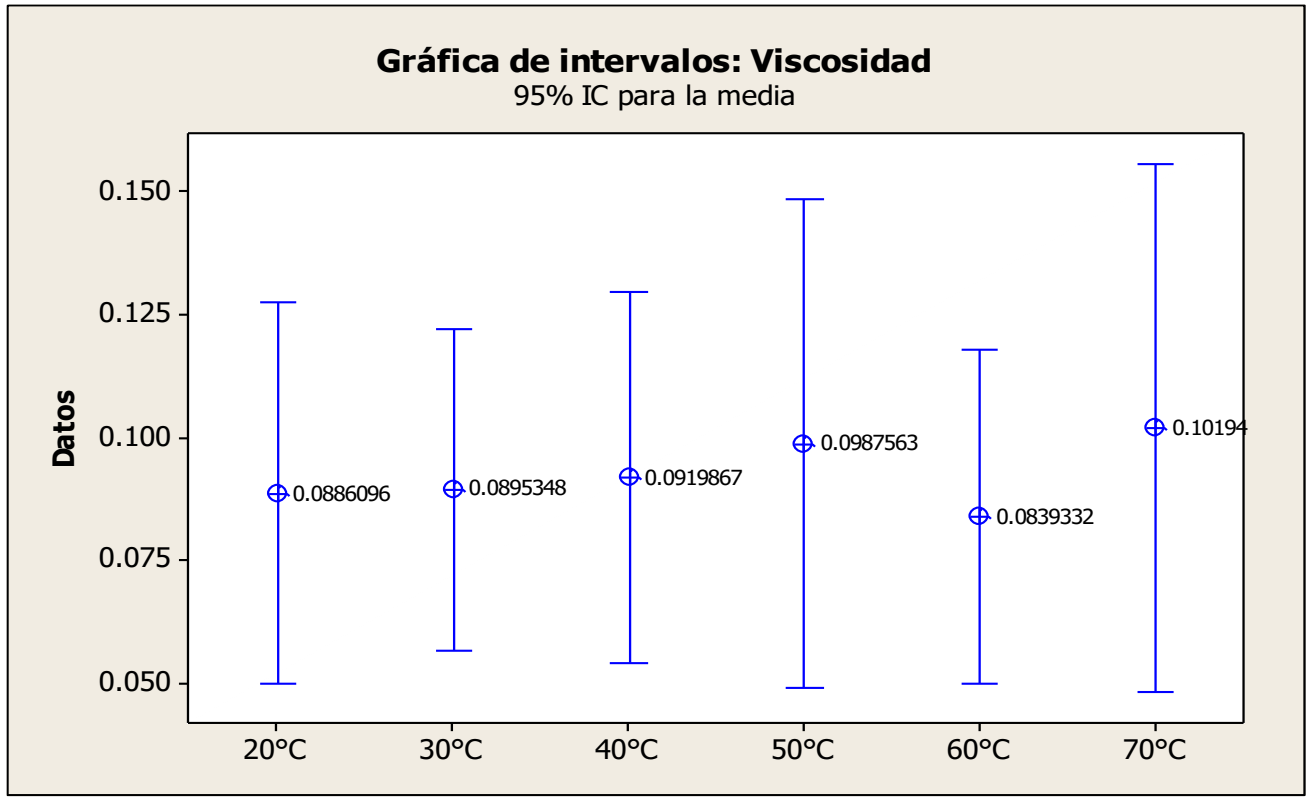

Alvarado (1992) determinó la viscosidad de jugos filtrados de frutas para un intervalo de temperatura de 10 a $80^{\circ} \mathrm{C}$ y calculó la energía de activación para el jugo de babaco (papaya o lechosa) comprendida en $23.7 \mathrm{~kJ} / \mathrm{mol}$. En el presente estudio, mediante el modelo de Herschel Bulkley se calculó la energía de activación para el Néctar mix papaya-piña, en el intervalo de 20 a $70^{\circ} \mathrm{C}$, resultando $0.4376 \mathrm{KJ} / \mathrm{mol}$ en promedio

Figura 12. Energía de activación de Herschel Bulkley

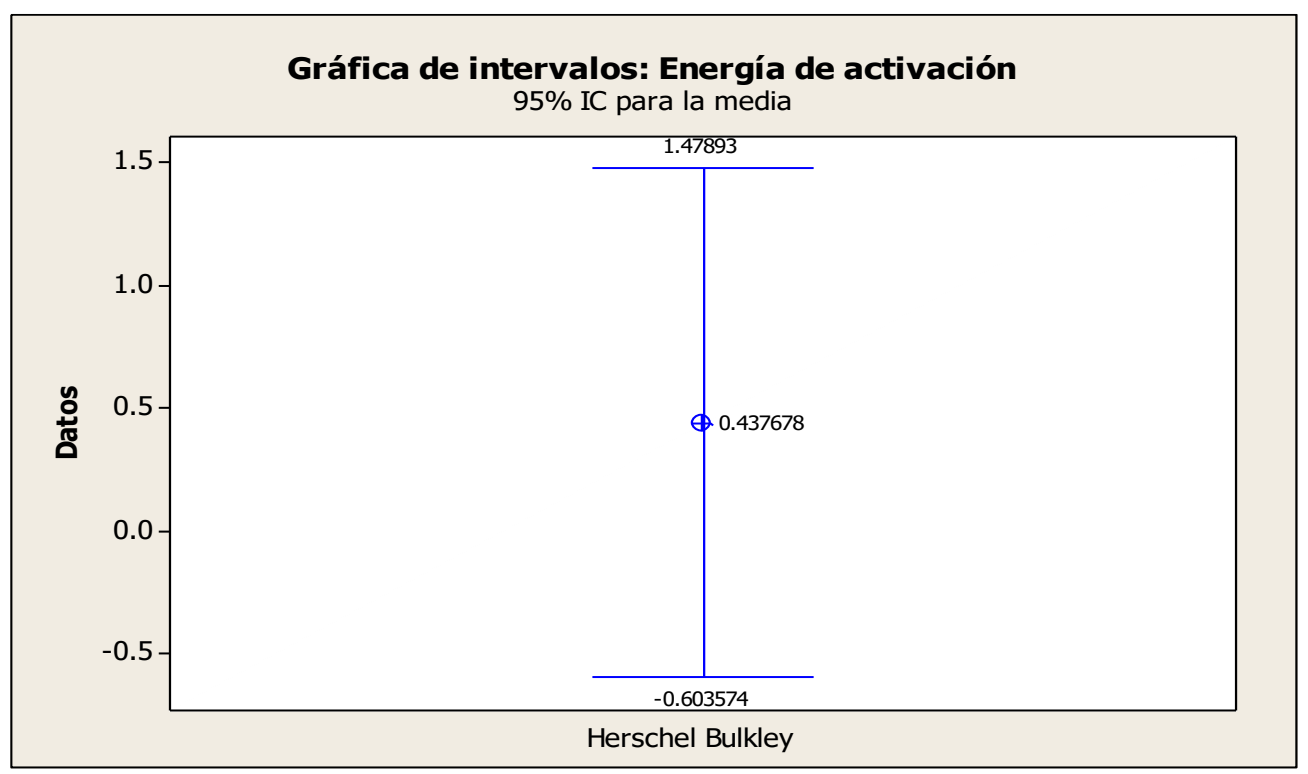




\section{Responsabilidad ética de acuerdo a los reglamentos vigentes}

Yo investigador me responsabilizo de los resultados obtenidos y la información obtenida de otros autores mencionados en la literatura afín

\section{CONCLUSIONES}

Se concluye que el Néctar mix papaya - piña presenta comportamiento reológico de un fluido plástico real, siendo el modelo de Herschel Bulkley, dado por la ecuación: $\tau=\tau_{0}+(\mu \gamma)^{1 / m}$, con un umbral de esfuerzo $=0.5667 \mathrm{~Pa}$, índice de consistencia $=$ 0.2185 Pa.s ${ }^{\mathrm{n}}$ e índice de flujo $=0.6401$ a la temperatura de $20^{\circ} \mathrm{C}$, así como una viscosidad aparente $=0.0473$ Pa.s y energía de activación $=3.7439 \mathrm{KJ} / \mathrm{mol}$ para el rango de temperatura de 20 a $70{ }^{\circ} \mathrm{C}$ a una velocidad de corte de $5 \mathrm{~s}^{-1}$, y con los valores estadísticos: coeficiente de correlación $\mathrm{R}^{2}=0.999238$ y varianza $\mathrm{S}^{2}=0.00157717$ para un nivel de confianza del 95\%, el modelo de representación óptima para este tipo de fluido en referencia a los demás modelos estudiados en esta tesis. Los datos reológicos se obtuvieron para las temperaturas de $10,20,3040,50$ y $60^{\circ} \mathrm{C}$, analizados en el sistema de cilindros concéntricos del reómetro Rheolab QC, hallándose una tendencia creciente y cóncava hacia abajo en los reogramas de esfuerzo y velocidad de corte, los cuales representan las características reológicas inherentes de Néctar Mix Papaya-Piña. Se determinó que para los rangos de temperatura trabajados, el índice de comportamiento de flujo presenta valores menores a $1(\mathrm{n}<0.5)$, y un índice de consistencia promedio de Herschel Bulkley 0.554867 Pa.s ${ }^{\mathrm{n}}$, características de un fluido no newtoniano con comportamiento plástico real, lo cual queda complementado con la verificación de la disminución de la viscosidad aparente [0.244012 - 0.0461511$]$ Pa.s a medida que se incrementa la velocidad de corte [ 5 - 100] s ${ }^{-1}$. Para el intervalo de temperatura $[20-50]{ }^{\circ} \mathrm{C}$ la viscosidad aparente muestra una un incremento en la viscosidad, esto debido a posibles formaciones de cristalinas en la estructura del fluido y para el intervalo de $[50-70]{ }^{\circ} \mathrm{C}$ el zumo presenta un comportamiento diferente, con disminución de la viscosidad aparente a $\operatorname{los} 60^{\circ} \mathrm{C}$, ocasionado por posibles cambios en la estructura cristalina y a un aumento significativo en la energía cinética de las partículas del fluido. 


\section{RECOMENDACIONES}

- Extender el estudio reológico de néctares con frutos de la zona para darle mayor valor agregado a sus subproductos.

- Extender el estudio reológico a nuevas formulaciones de néctares variando las concentraciones de sus componentes.

- Extender el estudio orientado a los cambios en la estructura interna que afecten a las mediciones del esfuerzo y velocidad de corte, principalmente alrededor de $\operatorname{los} 50^{\circ} \mathrm{C}$ en donde el fluido comienza a presentar posibles cambios en su estructura interna.

\section{REFENCIAS BIBLIOGRAFICAS}

Barnes, H. A. (2000). Non-Newtonian Flow in the Process Industries. RP Chhabra and JF Richardson, Butterworth-Heinnemann, Oxford, 1999, pp. xiii+ 436, \&35. Journal of Non-Newtonian Fluid Mechanics, 92(1), 106-106.

Canovas, G. B., \& Peleg, M. (1983). Flow parameters of selected commercial semiliquid food products. Journal of Texture Studies, 14(3), 213-234.

Cárdenas, J. C., López, O. J., \& Pinto, K. T. (2011). Estudio reológico de los fluidos viscoelásticos surfactantes utilizados en operaciones de fracturamiento hidráulico. Revista Fuentes, 9(1).

Carrasco Venegas, Luis (2011). Fenómenos de Transporte ( $2^{\circ}$ ed.) Editorial Macro, Perú: Lima.

Carrasco Venegas, Luis; Castañeda Pérez, Luz; Altamirano Oncoy, Karina (2014).

Modelos de Viscosidad de Fluidos No Newtonianos; Facultad de Ingeniería Química de la Universidad Nacional del Callao, Perú: Callao.

Carrasco Venegas, L., Castañeda Pérez, L., \& Altamirano Oncoy, K. (2015). Determinación de los parámetros reológicos de un champú con el modelo de Carreu-Yasuda.

Chhabra, R. P., \& Richardson, J. F. (1999). Non-Newtonian flow in the process industries: fundamentals and engineering applications. Butterworth-Heinemann.

Franco Matos, C. E., \& Sanchez Casas, C. C. (2018). Determinación de los modelos reológicos asociados al zumo de piña.

Ibarrola, E. L. (2009). Introducción a los fluidos no newtonianos. Cátedra de Mecánica de fluidos. UnCor. 
Javier Daza, N. R. (2014). Elaboración y evaluación reológica de mermelada de piña (Ananas comosus).

J.R. Hermida Bun (2000). Fundamentos de Ingeniería de Procesos Agroalimentarios.

López, L. T., \& Miranda, L. S. (2018). Efecto de la viscosidad de una suspension en la clasificación de hidrociclones.

Lozada Carbajal, M. A. (2007). Extracción y caracterización reológica de polisacáridos tipo pectina de la cáscara de tuna (Opuntia spp.).

Magerramov, M. A., Abdulagatov, A. I., Abdulagatov, I. M., \& Azizov, N. D. (2007). Viscosity of tangerine and lemon juices as a function of temperature and concentration. International journal of food science \& technology, 42(7), 804818.

Méndez, A. R., \& Ramos, O. A. N. (2008). Comportamiento reológico de un fluido. Universidad del Mar. Oaxaca, México.

Molina Rodriguez, E. D., \& Rojas Quispe, R. M. (2018). Modelos reologicos asociados a la sangre.

Monsalve, A. (2010). Reología, la ciencia que estudia el movimiento de fluidos. Revista Remetallica, (18).

Montalván Torres, G. (2018). Reogramas de modelos de fluidos no Newtonianos.

Mott, Robert L. (2006). Mecánica de Fluidos (6ed). Ed. Pearson Educación. México.

Pérez Alferes, p. J. (2015). Comportamiento reológico de la pulpa tamizada de copoazú (Theobroma grandiflorum).

Pérez-Trejo, L., Sánchez, A. F. M., \& Mercado, A. P. (2010). Determinación de la viscosidad de fluidos newtonianos y no newtonianos (una revisión del viscosímetro de Couette). Latin-American Journal of Physics Education, 4(1), 36.

Quintero, C., Duque, C., \& Giraldo, G. (2012). Evaluation of the viscosity and the color of common mango pulp (Mangifera indica L.) enzymatically treated. Revista Temas Agrarios, 17(2), 66-76.

Ramírez Navas, Juan, (2006). Fundamentos de Reología de los Alimentos; Ed. JSR ebooks, Colombia: Cali.

Rao, M. A., Rizvi, S. S., Datta, A. K., \& Ahmed, J. (2014). Engineering properties of foods. CRC press. 
Rauendaal C. (1988). Mixing in Polymer Processing, Munich, Hanser.

Rojas, O. (1999). Introducción a la Reología. Cuaderno FIRP, 520.

Saravacos, G. D. (1970). Effect of temperature on viscosity of fruit juices and purees. Journal of Food Science, 35(2), 122-125.

Sharma, S. K., \& Mulvaney, S. J. (2003). Ingeniería de alimentos: operaciones unitarias y prácticas de laboratorio (No. 664 S32). Saravacos, G. D., \& Maroulis, Z. B. (2001). Transport properties of foods. CRC Press.

Soler Manuel, M. A. (1976). Hidrodinámica de los fluidos no-newtonianos: aplicación al derrame en un medio poroso. Universitat Politècnica de Catalunya.

Steffe, J. F. (1996). Rheological methods in food process engineering. Freeman press. 